\title{
Genome update: the 1000th genome - a cautionary tale
}

There are now more than 1000 sequenced prokaryotic genomes deposited in public databases and available for analysis. Currently, although the sequence databases GenBank, DNA Database of Japan and EMBL are synchronized continually, there are slight differences in content at the genomes level for a variety of logistical reasons, including differences in format and loading errors, such as those caused by file transfer protocol interruptions. This means that the 1000th genome will be different in the various databases. Some of the data on the highly accessed web pages are inaccurate, leading to false conclusions for example about the largest bacterial genome sequenced. Biological diversity is far greater than many have thought. For example, analysis of multiple Escherichia coli genomes has led to an estimate of around 45000 gene families - more genes than are recognized in the human genome. Moreover, of the 1000 genomes available, not a single protein is conserved across all genomes.

Excluding the members of the Archaea, only a total of four genes are conserved in all bacteria: two protein genes and two RNA genes.

\section{Introduction}

Sometime in October or November 2009 , depending on which database is consulted, the 1000th prokaryotic genome sequence was completed. This landmark followed within 15 years of the sequencing of the first bacterial genomes, Haemophilus influenzae and Mycoplasma genitalium (Fleischmann et al., 1995; Fraser et al., 1995). The generation of these and other early genome sequences were costly in terms of both time and money, and the efforts were justly rewarded by patents and publications in the journals Science and Nature. Much has changed since then, not least by breathtaking technical innovations in sequencing procedures (the end of which is not yet in sight) supported by steadily increasing computer power and constantly improving software (Ansorge, 2009; Kyrpides, 2009). A bacterial genome can now be completely sequenced, assembled and annotated in less than $24 \mathrm{~h}$ (Flicek \& Birney, 2009; Reeves et al., 2009). The necessary final steps required for full closure of all the gaps and quality assessment of the annotation are, however, still time consuming, although it is possible, even with the current technology, to completely assemble a bacterial genome based on a single run from a 'next generation' machine (Tauch et al., 2008). The imminent 'third generation' sequencing machines are promising vast improvements and one can envision a time in the not-too-distant future when it will be routine to sequence and annotate several bacterial genomes before the morning tea break.

As every microbiologist knows, there has been a dramatic increase in the number of sequenced microbial genomes over the past decade; this is illustrated for prokaryotic genomes in Fig. 1. The exponential growth in the number of finished genomes per year seemed to reach a peak of approximately 180 per year by 2007 , and then declined slightly for 2008 and 2009 . Have we reached a 'stationary phase' of bacterial genome sequencing? There are possible explanations for this decline, and one key observation is that the number of unfinished genomes deposited in GenBank now is larger than the number of complete genomes, as the production of a 'rough draft' has become relatively inexpensive. As of early January 2010, there are 1024 complete gen- omes listed at NCBI and more than twice as many genomes (2307) listed as 'in progress'; the Genomes Online Database (GOLD) web pages (see below and link in Table 1) boast more than 6400 microbial genome sequencing projects.

\section{The 1000th genome(s)}

In principle, there should be a list where one could go to find the 1000th genome; however, as several genomes are processed and submitted on an almost daily basis to databases, determining the 1000th genome is not as easy as might appear at first. Table 1 lists the set of genomes for the various databases. According to GOLD (which is perhaps one of the best centralized locations for keeping track of this), the 1000th genome is an archaeon: Methanocaldococcus vulcanius strain M7 (accession CP001787), sequenced by the Joint Genomes Institute (JGI). The honour of being number 1000 is not as clear for the other databases. For the DNA Database of Japan (DDBJ), there are three candidate genomes which were all added on the same day; these have also been sequenced by the JGI. One of these is another Methanocaldococcus (this time $M$. fervens), which has not yet been published (CP001696). The other two genomes are part of the Genomic Encyclopedia of Bacteria and Archaea (GEBA) project (Wu et al., 2009). Kangiella koreensis is a member of the phylum Gammaproteobacteria, belonging to the order Oceanospirillales; this genome is from the type strain (DSM 16069), isolated from a beach in South Korea (Han et al., 2009). Slackia heliotrinireducens DSM 20476 is a member of the phylum Actinobacteria, belonging to the uncharacterized family Coriobacteriaceae (Pukall et al., 2009).

The '1000th genome' from EMBL is a set of Acetobacter pasteurianus gen- 


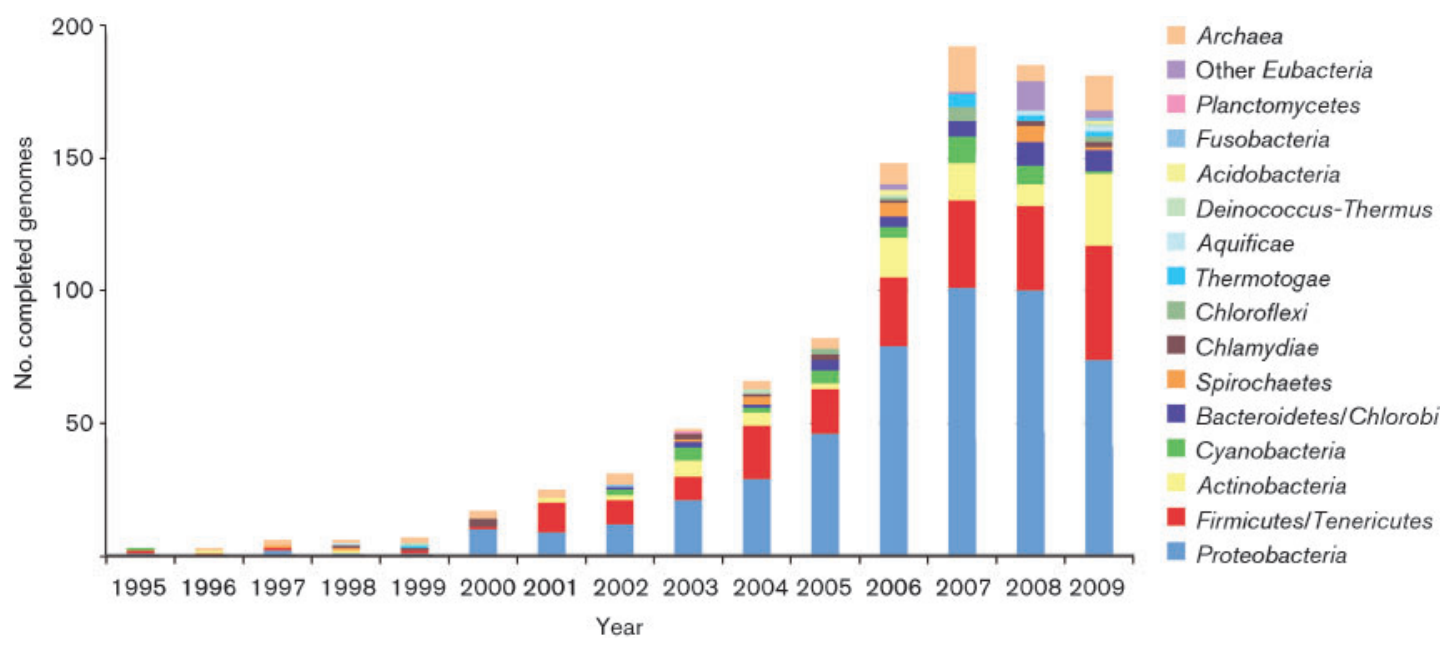

Fig. 1. Increase in the number of genomes completed per year separated by bacterial phylum. Data source: NCBI, complete genomes (http://www.ncbi.nlm.nih.gov/genomes/lproks.cgi).

omes from eight different strains. Acetobacter species have been used historically for making vinegar and are known to be quite variable in terms of their genome content, due to a large number of transposons (Azuma et al., 2009). Although the eight $A$. pasteurianus strains are also present in DDBJ and GenBank, only one sequence is found in the 'complete genomes' list on the NCBI web pages, and the other seven genomes, although finished to one contiguous piece, are listed as 'in progress' more than 6 months after being deposited in the International Nucleotide Sequence Database.

Finally, we have listed (Table 1) a set of seven genomes deposited in GenBank around the same time. At one stage, Vibrio sp. isolate Ex25, was listed as the 1000th genome on the NCBI pages, although with time, as some older genomes have been removed and the lists have been updated, the 1000th position has moved a bit, so a range of genomes is given. Of the seven genomes in the list, five have at least one publication associated with them at the time of writing; Vibrio sp. Ex25 (CP001805) and Staphylococcus aureus ED98 (CP001781) genomes have not been described in a publication. Three of the genomes are from the GEBA project (Wu et al., 2009):
Rhodothermus marinus strain R-10T is a member of the phylum Bacteroidetes and was isolated from hot springs off the coast of Iceland (Nolan et al., 2009); Gordonia bronchialis DSM 43247 is a member of the phylum Actinobacteria, isolated from the sputum of a woman with diseased lungs; and Haliangium ochraceum DSM 14365 is a halophilic deltaproteobacterium isolated from coastal sand in Japan. The Blattabacterium genome sequenced, which is from a cockroach endosymbiotic strain, belongs to the class Flavobacteria in the phylum Bacteroidetes; the newly sequenced genome shows evolutionary convergence with gammaproteobacterium endosymbionts (LópezSánchez et al., 2009). Finally, Comamonas testosteroni strain CNB-2 was isolated from soil contaminated with 4-chloronitrobenzene and can grow on this pollutant using it as its sole carbon and nitrogen source ( $\mathrm{Ma}$ et al., 2009). This betaproteobacterium is a member of the order Burkholderiales, and was given its name from its ability to metabolize testosterone.

Of the 1000 prokaryotic genomes sequenced so far, only $7 \%$ are archaea, with the rest $(93 \%)$ being bacteria. Whether this ratio is truly reflective of the relative proportions in the environment is doubtful. Within the bac- teria, members of the phyla Proteobacteria and Firmicutes make up the majority of the completed genome sequences, and many of the new genomes sequenced each year are found within these two phyla. Together, these account for $72 \%$ (of 930) of bacterial genomes, with 489 (52\%) proteobacteria and 187 (20\%) firmicute genomes (GOLD data). A similar skewed distribution is observed for the archaeal genomes, where the majority are from the phylum Euryarchaeota (63\%); with nearly all the rest coming from the phylum Crenarchaeota (31\%). The phylum Nanoarchaoeta has only a single sequenced genome. Such an uneven distribution in available sequences has consequences for the chance that a particular query sequence will identify similarities in the database: when a query sequence from a proteobacterial genome is used to search the microbial database using BLAST, the chance is much higher that a hit will be found than, for instance, searching with a nanoarchaoeta sequence. Since the E-value reported by BLAST is based on the expected background noise, and is based on the assumption that the sequences within the database are random (which obviously is not true in this case), the results should be interpreted with caution when the 'best hit' of a 
Table 1. Summary of the published genomes discussed in this article

The accession number for each chromosome used in the International Nucleotide Sequence Database (INSD; www.insdc.org) is the same for the DDBJ, EMBL and GenBank databases.

\begin{tabular}{|c|c|c|c|c|c|c|c|}
\hline Strain & Taxonomic phylum & $\begin{array}{c}\text { Length } \\
\text { (bp) }\end{array}$ & $\begin{array}{c}\text { AT content } \\
(\%)\end{array}$ & $\begin{array}{c}\text { No. } \\
\text { genes }\end{array}$ & $\begin{array}{l}\text { No. } \\
\text { rRNAs }\end{array}$ & $\begin{array}{l}\text { No. } \\
\text { tRNAs }\end{array}$ & $\begin{array}{c}\text { Accession } \\
\text { no. }\end{array}$ \\
\hline \multicolumn{8}{|l|}{ GOLD } \\
\hline $\begin{array}{l}\text { Methanocaldococcus vulcanius M7 } \\
\text { DDBJ }^{\star}\end{array}$ & Euryarchaeota & 1761737 & 68.4 & 1742 & 2 & 37 & CP001787 \\
\hline Slackia heliotrinireducens DSM 20476 & Actinobacteria & 3165038 & 39.8 & 2765 & 2 & 48 & СР001684 \\
\hline Methanocaldococcus fervens AG86 & Euryarchaeota & 1507251 & 67.8 & 1581 & 2 & 37 & СР001696 \\
\hline \multicolumn{8}{|l|}{ EMBL $\dagger$} \\
\hline Acetobacter pasteurianus IFO 3283-01 & Alphaproteobacteria & 3340249 & 46.9 & 3050 & 5 & 57 & GPID 31129 \\
\hline A. pasteurianus IFO 3283-01 & Alphaproteobacteria & 2907495 & 50.0 & 2628 & 5 & 57 & AP011121 \\
\hline A. pasteurianus IFO $3283-01-42 \mathrm{C}$ & Alphaproteobacteria & 2815241 & 46.9 & 2562 & 4 & 54 & AP011163 \\
\hline A. pasteurianus IFO 3283-03 & Alphaproteobacteria & 2907287 & 50.0 & 2627 & 5 & 57 & AP011128 \\
\hline A. pasteurianus IFO 3283-07 & Alphaproteobacteria & 2906044 & 50.0 & 2626 & 5 & 57 & AP011135 \\
\hline A. pasteurianus IFO $3283-22$ & Alphaproteobacteria & 2907267 & 50.0 & 2627 & 5 & 57 & AP011142 \\
\hline A. pasteurianus IFO 3283-26 & Alphaproteobacteria & 2907309 & 50.0 & 2627 & 5 & 57 & AP011149 \\
\hline A. pasteurianus IFO 3283-32 & Alphaproteobacteria & 2904642 & 50.0 & 2625 & 5 & 57 & AP011156 \\
\hline A. pasteurianus IFO 3283-12 & Alphaproteobacteria & 2904624 & 50.0 & 2625 & 5 & 57 & AP011170 \\
\hline \multicolumn{8}{|l|}{ GenBank $₫$} \\
\hline Rhodothermus marinus DSM 4252 & Bacteroidetes & 3386737 & 35.7 & 2863 & 1 & 45 & СР001807 \\
\hline Vibrio sp. Ex25 & Gammaproteobacteria & 5089025 & 55.1 & 4518 & 11 & 124 & СР001805 \\
\hline Blattabacterium sp. & Bacteroidetes & 636850 & 72.9 & 587 & 1 & 34 & СР001487 \\
\hline Comamonas testosteroni CNB-2 & Betaproteobacteria & 5373643 & 38.6 & 4803 & 3 & 79 & СР001220 \\
\hline Gordonia bronchialis DSM 43247 & Actinobacteria & 5290012 & 33.0 & 4696 & 2 & 49 & СР001802 \\
\hline Haliangium ochraceum DSM 14365 & Deltaproteobacteria & 9446314 & 30.5 & 6719 & 2 & 46 & СР001804 \\
\hline Staphylococcus aureus ED98 & Firmicutes & 2847542 & 67.2 & 2689 & 5 & 61 & СР001781 \\
\hline
\end{tabular}

${ }^{*}$ DDBJ, http://gib.genes.nig.ac.jp

$\dagger$ EMBL, http://www.ebi.ac.uk/genomes/bacteria.html

\#GPID is NCBI’s Genome Project ID, a genome identifier unique to NCBI and not part of the INSD. The GPID contains a combination of the main chromosome plus six plasmids. The data shown in the table for the other A. pasteurianus genomes are for the main chromosome only, although each of these genomes also contains six plasmids in addition to the main chromosome.

$\S$ GenBank (also referred to as 'NCBI web pages'), http://www.ncbi.nlm.nih.gov/genomes/lproks.cgi

nanoarchaoeta gene is to a proteobacteria gene.

There are 612 unique species represented within the 1000 genomes. Thus, for many species there are multiple genomes sequenced, usually from multiple strains, and if this redundancy is removed from the data, the coverage of genomes per phylum does not significantly change the distribution; this is true for data from both the GOLD and the NCBI databases. The species for which the most genomes are completed is currently Escherichia coli, for which 32 genomes have been completely sequenced. Much of the emphasis so far in bacterial genomics is on (human) pathogenic bacteria, as reflected in the species for which multiple strains have been sequenced, although it is likely that these represent only a fraction of the true bacterial diversity on Earth.

\section{Numerical characteristics of sequenced genomes}

One thousand bacterial genomes represent a few billion nucleotides and a few million genes. To analyse such a wealth of information would require immense computing time, so that the 2000th genome would probably be sequenced prior to any in-depth analysis of the first 1000 being com- pleted. Therefore, we have focussed our efforts on a few basic analyses, details of which are described elsewhere (Ussery et al., 2009). A striking difference is noted in the variation of genome size within phyla: whereas members of the phyla Proteobacteria, Cyanobacteria, Actinobacteria, Chloroflexi and Bacteroidetes/Chlorobi display a wide variation in genome size, those of the phyla Thermotoga, Fusobacteria and Aquificae show little differences in size within the same phylum. (A box and whiskers plot is provided in Supplementary Figure S1, available in Microbiology Online, which is an updated version of Fig. 1 from Ussery \& Hallin, 2004a.) Some of this conservation in genome size could be 
due to the restricted number of genomes available, although three acidobacteria genomes already display a large variation in genome size variation. Compared with the wide variety in genome size within bacterial phyla, especially when outliers are considered, the variation between phyla is less dramatic. As expected, intracellular parasites, such as members of the phyla Chlamydiae and Thermotogae, typically have small genomes (1-2 Mbp), whereas those of the phyla Proteobacteria and Chloroflexi usually have larger genomes $(\sim 4 \mathrm{Mb}$ on average). The record holders are currently Sorangium cellulosum strain 'So ce 56', a deltaproteobacterium that can produce several bioactive compounds, with a genome of $13 \mathrm{Mbp}$, and Candidatus Hodgkinia cicadicola, an alphaproteobacterium, with a genome of only $143 \mathrm{kbp}$. Note: the NCBI pages list two larger bacterial genomes, but closer inspection shows that the sizes of these genomes are much smaller than indicated. The GOLD web pages list the largest genome as Solibacter usitatus Ellin6076, at $9.965 \mathrm{Mbp}$, and the second largest as Mycoplasma gallisepticum $\mathrm{R}$, which is only $0.996 \mathrm{Mbp}$ (apparently this field is sorted by text rather than numbers). Currently, both EMBL and DDBJ cannot be sorted by genome size on their genome web pages. Thus, the size range between the largest and smallest bacterial genome is approaching 100fold.

It is also interesting to observe how the average length of genomes being sequenced has increased over the years. Until 2001, the average genome being sequenced was $2.4 \mathrm{Mbp}$, after that, the average increased to $3.7 \mathrm{Mbp}$ - a shift that probably reflects the improved technology in generating, handling and assembling large genomes, and the decreased costs so that size is now less of a limiting factor.

A second characteristic given by a numerical value is GC content, which again varies between phyla, as discussed in a previous Genome Update article (Ussery \& Hallin, 2004b; see Supplementary Figure S2 for an updated version of the box-and-whis- kers plot). The phylum Proteobacteria contains genomes with widely varying GC content whereas members of the phyla Actinobacteria and Firmicutes have a much narrower GC content distribution. Members of other phyla commonly have a GC content somewhere between 40 and $50 \%$, with the exception of phyla for which only a few genomes have so far been sequenced, in which case a skew due to limited data cannot be ruled out.

Superficially, there seems to be a correlation between GC content and genome size. For instance, using the NCBI data, an increase in GC content of roughly $3.7 \%$ GC per Mbp can be observed. Examining the phyla for which we have the most genomes, this tendency was also seen among the proteobacteria (an increase of $5 \%$ GC per $\mathrm{Mbp}$ ) and the actinobacteria (an increase of $2 \%$ per Mbp). However, a significant relationship was not found among the firmicutes or the archaea. The observed relationship between GC content and genome size, within and between some phyla, could be the result of differences in lifestyle. Many intracellular bacteria with small genomes also have a low GC content (Moran \& Baumann, 2000). Since organisms that encounter multiple variable conditions would also require more genes to cope with this variability, genome size could also correlate with ecological niche, so that genome size correlates to some extent with gene content, assuming a more or less invariable gene density.

Fig. 2 shows the variation of gene density per genome among the phyla. As is evident, the gene density does not vary much for the majority of genomes, with a mean of 918 genes per Mbp. The gene density is also quite uniform within each phylum; the only one that deviates from this to any extent is that of the phylum Chloroflexi. Most of the genomes within this phylum have a density of around 770 genes per Mbp; however, there are three genomes in the phylum Dehalococcoides that have densities around 1050 genes per Mbp. These genomes are also quite short, with an average length of 1.4 Mbp.

Fig. 2. Box-and-whiskers plot of gene density of prokaryotes per phylum separated by bacterial phylum. Data are from NCBI. 


\section{Comparison of gene content}

With the release of the complete genome of $M$. genitalium, the quest to identify the prokaryotic genome with the smallest possible number of genes began (Fraser et al., 1995). Some endosymbiotic bacteria have lost a large fraction of their genes as their host provides them with essential biomolecules (Moya et al., 2009). This particularly applies to intracellular bacteria living in symbiosis with an insect host which can have severely reduced genomes specialized to produce nutrients that are not included in the host's diet (McCutcheon et al., 2009). The publication of the extremely small Carsinella rudii genome, with only 182 predicted protein-coding genes (Nakabachi et al., 2006), started a discussion on whether or not to accept this as the genome of a true living cell: it was argued that genes coding for a complete machinery for DNA replication, transcription and translation, and a (simplified) metabolic network for energy production would have to be present, but a number of essential genes for these processes are missing in $C$. rudii (Tamames et al., 2007). However, there is no fundamental biological difference between a symbiont depending on its host, for instance for a particular amino acid or an enzyme needed to supercoil its DNA; the requirement to independently produce its own DNA, RNA and proteins would not be met either way. We therefore consider that the genome of $C$. rudii represents that of a prokaryotic cell as validly as any other. The even smaller genome of Hodgkinia cicadicola (which, as an exception to the rule, has a very small genome but a high GC content) has specialized to produce vitamin B12 for its insect host, for which it reserves $7 \%$ of its proteome that in total only encodes 169 proteins (McCutcheon et al., 2009).

Another goal of comparative genomics has been to recognize those proteincoding genes that are conserved in all prokaryotic genomes, as these would represent the ultimate core genome of bacteria. The outcome of such ana- lyses obviously depends on the criteria applied to gene definition, to orthologous inclusion as well as on the decision to include reduced symbiont genomes. When the genomes of $H$. influenzae and $M$. genitalium were compared in 1996, a total of 256 genes were recognized as conserved (Mushegian \& Koonin, 1996) and this number was reduced to 179 genes when five endosymbiont genomes were added (Gil et al., 2003). Adding Rickettsia prowazekii and Chlamydia trachomatis genomes reduced the number to 156 conserved orthologous genes (Klasson \& Andersson, 2004). This number is certainly expected to further decrease if all 1000 genomes are included (it is acknowledged here that this analysis ignores functionally equivalent genes that lack sequence similarity, as noted by Gil et al., 2004). Only 31 proteins were found to be conserved across 191 species (Ciccarelli et al., 2006). How many proteins do we find conserved across all bacteria? The results might be surprising to some.

For reasons of computational simplicity, we took the described genes of the two smallest symbiont genomes ( $C$. rudii and $H$. cicadicola) as a starting point, and compared these with the two largest genomes currently available per bacterial phylum, including the Archaea; this resulted in a set of 32 genomes from 16 different phyla. From this artificial subset of 32 genomes, a core genome analysis was performed using a similarity cutoff of at least $50 \%$ identity at the protein level, over at least $50 \%$ of the longest gene in reciprocal pairwise analysis (as described by Tettelin et al., 2005). For the entire set of 1000 genomes, not a single protein-coding gene was found to be conserved. When we exclude members of the Archaea, two protein-coding genes were conserved, these were the translation elongation factor EF-Tu and the ribosomal protein S12. Furthermore, the $16 \mathrm{~S}$ and $23 \mathrm{~S}$ rRNAs were found to be conserved.

Finally, we compared the first two genomes sequenced, those of $M$. genitalium and $H$. influenzae, with the genomes listed in Table 1. As expected, all genomes of strains in the genus Methanocaldococcus were extremely similar, sharing $>98 \%$ of their genes in any pairwise comparison (see Supplementary Figure S3). The three gammaproteobacteria (Vibrio sp., $H$. influenzae and $K$. koreensis) share $10-15 \%$ of their genes, but there are only nine genes shared between Methanocaldococcus and $H$. influenzae $\mathrm{Rd}$, and only two genes are shared between Methanocaldococcus and M. genitalium. All other combinations of genomes share between 0.2 and $6 \%$ of their genes. The conclusion is that a minimal gene set required for life depends, as does everything else in biology, on the context. There is certainly a conserved set of gene functions necessary for life, but this does not necessarily correlate with a set of conserved gene sequences.

\section{Conclusion}

The number of sequenced genomes is quickly expanding, and although there is an International Nucleotide Sequence Database, it appears to not be working so well for genome sequences. Currently, the number of genomes available is a bit more than 1000, but none of the three databases (DDBJ, EMBL or GenBank) contain all of the genomes. Further, some of the web pages contain inaccurate information. There is a real need for a clearly visible place for microbiologists to go to obtain up-to-date information about genome sequences. Currently, there are three different formats for the three different databases, and unfortunately these three databases are not synchronized on a regular basis. Further, rules about when a genome is 'fully sequenced' should be further explored (Kyrpides, 2009; Chain et al., 2009). This is essential for a full and reliable comparison of the vast genomic resources available. What is already obvious, though, is an incredible diversity within these genomes, with essentially no genes being fully conserved across all prokaryotes, using the same standard criteria as used by others (Tettelin et al., 2005). Perhaps it is best to consider using alternative methods of defining gene families, in some way based more on conserved function. 


\section{Supplementary data}

Supplementary figures showing boxand-whiskers plots of the genome size of prokaryotes per phylum and of the GC content of prokaryotes per phylum, and of the BLAST matrix of the genomes from Table 1 are available with the online version of this paper.

\section{Karin Lagesen,, ${ }^{1,2}$ Dave W. Ussery ${ }^{1}$ and Trudy M. Wassenaar ${ }^{1,3}$ \\ ${ }^{1}$ Center for Biological Sequence Analysis, Department of Systems Biology, The Technical University of Denmark, 2800 Lyngby, Denmark \\ ${ }^{2}$ Centre for Molecular Biology and Neuroscience, Institute of Medical Microbiology, Oslo University Hospital, Rikshospitalet, NO-0027, Oslo, Norway, and Department of Informatics, University of Oslo, PO Box 1080 Blindern, NO-0316, Oslo, Norway \\ ${ }^{3}$ Molecular Microbiology and Genomics Consultants, Zotzenheim, Germany \\ Correspondence: Dave W. Ussery (dave@cbs.dtu.dk)}

Ansorge, W. J. (2009). Next-generation DNA sequencing techniques. Nat Biotechnol 25, 195-203.

Azuma, Y., Hosoyama, A., Matsutani, M., Furuya, N., Horikawa, H., Harada, T., Hirakawa, H., Kuhara, S., Matsushita, K. \& other authors (2009). Whole-genome analyses reveal genetic instability of Acetobacter pasteurianus. Nucleic Acids Res 37, 5768-5783.

Chain, P. S., Grafham, D. V., Fulton, R. S., Fitzgerald, M. G., Hostetler, J., Muzny, D., Ali, J., Birren, B., Bruce, D. C. \& other authors (2009). Genomics. Genome project standards in a new era of sequencing. Science 326, 236-267.

Ciccarelli, F. D., Doerks, T., von Mering, C., Creevy, C. J., Snel, B. \& Bork, P. (2006). Toward automatic reconstruction of a highly resolved tree of life. Science 311, 1283-1287.

Fleischmann, R. D., Adams, M. D., White, O., Clayton, R. A., Kirkness, E. F., Kerlavage, A. R., Bult, C. J., Tomb, J. F., Dougherty, B. A. \& other authors (1995). Whole-genome random sequencing and assembly of Haemophilus influenzae Rd. Science 269, 496-512.

Flicek, P. \& Birney, E. (2009). Sense from sequence reads: methods for alignment and assembly. Nat Methods 6 (Suppl. 11), S6-S12.
Fraser, C. M., Gocayne, J. D., White, O., Adams, M. D., Clayton, R. A., Fleischmann, R. D., Bult, C. J., Kerlavage, A. R., Sutton, G. \& other authors (1995). The minimal gene complement of Mycoplasma genitalium. Science 270, 397-403.

Gil, R., Silva, F. J., Zientz, E., Delmotte, F., González-Candelas, F., Latorre, A., Rausell, C., Kamerbeek, J., Gadau, J. \& other authors (2003). The genome sequence of Blochmannia floridanus: comparative analysis of reduced genomes. Proc Natl Acad Sci U S A 100, 93889393.

Gil, R., Silva, F. J., Peretó, J. \& Moya, A. (2004). Determination of the core of a minimal bacterial gene set. Microbiol Mol Biol Rev 68, 518-537.

Han, C., Sikorski, J., Lapidus, A., Nolan, M., Del Rio, T. G., Tice, H., Cheng, J.-F., Lucas, S., Chen, F. \& other authors (2009). Complete genome sequence of Kangiella koreensis type strain $\left(\mathrm{SW}-125^{\mathrm{T}}\right)$. Stand Genomic Sci 1, 3. doi:10.4056/sigs.36635.

Klasson, L. \& Andersson, S. G. (2004). Evolution of minimal-gene-sets in hostdependent bacteria. Trends Microbiol 12, 37-43.

Kyrpides, N. C. (2009). Fifteen years of microbial genomics: meeting the challenges and fulfilling the dream. Nat Biotechnol 27, 627-632.

López-Sánchez, M. J., Neef, A., Peretó, J., Patiño-Navarrete, R., Pignatelli, M., Latorre, A. \& Moya, A. (2009). Evolutionary convergence and nitrogen metabolism in Blattabacterium strain Bge, primary endosymbiont of the cockroach Blattella germanica. PLoS Genet 5, e1000721.

Ma, Y. F., Zhang, Y., Zhang, J. Y., Chen, D. W., Zhu, Y., Zheng, H., Wang, S. Y., Jiang, C. Y., Zhao, G. P. \& Liu, S. J. (2009). The complete genome of Comamonas testosteroni reveals its genetic adaptations to changing environments Appl Environ Microbiol 75, 6812-6819.

McCutcheon, J. P., McDonald, B. R. \& Moran, N. A. (2009). Convergent evolution of metabolic roles in bacterial co-symbionts of insects. Proc Natl Acad Sci U S A 106, 15394-15399.

Moran, N. A. \& Baumann, P. (2000). Bacterial endosymbionts in animals. Curr Opin Microbiol 3, 270-275.

Moya, A., Gil, R. \& Latorre, A. (2009). The evolutionary history of symbiotic associations among bacteria and their animal hosts: a model. Clin Microbiol Infect 15 (Suppl. 1), 11-13.

Mushegian, A. R. \& Koonin, E. V. (1996). A minimal gene set for cellular life derived by comparison of complete bacterial genomes. Proc Natl Acad Sci U S A 93, 10268-10273.

Nakabachi, A., Yamashita, A., Toh, H., Ishikawa, H., Dunbar, H. E., Moran, N. A. \&
Hattori, M. (2006). The 160-kilobase genome of the bacterial endosymbiont Carsonella. Science 314, 267.

Nolan, M., Tindall, B. J., Pomrenke, H., Lapidus, A., Copeland, A., Del Rio, T. G., Lucas, S., Chen, F., Tice, H. \& other authors (2009). Complete genome sequence of Rhodothermus marinus type strain $\left(\mathrm{R}-10^{\mathrm{T}}\right)$. Stand Genomic Sci 1, 3. doi:10.4056/sigs.42644.

Pukall, R., Lapidus, A., Nolan, M., Copeland, A., Del Rio, T. G., Lucas, S., Chen, F., Tice, H., Cheng, J.-F. \& other authors (2009). Complete genome sequence of Slackia heliotrinireducens type strain $\left(\mathrm{RSH} 1^{\mathrm{T}}\right)$. Stand Genomic Sci $\mathbf{1}, 3$. doi:10.4056/sigs.37633.

Reeves, G. A., Talavera, D. \& Thornton, J. M. (2009). Genome and proteome annotation: organization, interpretation and integration. J R Soc Interface 6, 129-147.

Tamames, J., Gil, R., Latorre, A., Peretó, J., Silva, F. J. \& Moya, A. (2007). The frontier between cell and organelle: genome analysis of Candidatus Carsonella ruddii. BMC Evol Biol 7, 181.

Tauch, A., Schneider, J., Szczepanowski, R., Tilker, A., Viehoever, P., Gartemann, K. H., Arnold, W., Blom, J., Brinkrolf, K. \& other authors (2008). Ultrafast pyrosequencing of Corynebacterium kroppenstedtii DSM44385 revealed insights into the physiology of a lipophilic corynebacterium that lacks mycolic acids. J Biotechnol 136, 22-30.

Tettelin, H., Masignani, V., Cieslewicz, M. J., Donati, C., Medini, D., Ward, N. L., Angiuoli, S. V., Crabtree, J., Jones, A. L. \& other authors (2005). Genome analysis of multiple pathogenic isolates of Streptococcus agalactiae: implications for the microbial "pan-genome". Proc Natl Acad Sci U S A 102, 13950-13955.

Ussery, D. W. \& Hallin, P. F. (2004a). Genome update: length distributions of sequenced prokaryotic genomes. Microbiology 150, 513-516.

Ussery, D. W. \& Hallin, P. F. (2004b). Genome update: AT content in sequenced prokaryotic genomes. Microbiology 150, 749-752.

Ussery, D. W., Wassenaar, T. M. \& Borini, S. (2009). Computing for Comparative Microbial Genomics: Bioinformatics for Microbiologists. London, UK: Springer.

Wu, D., Hugenholtz, P., Mavromatis, K., Pukall, R., Dalin, E., Ivanova, N. N., Kunin, V., Goodwin, L., Wu, M. \& other authors (2009). A phylogeny-driven genomic encyclopaedia of Bacteria and Archaea. Nature 462, 1056-1060.

DOI 10.1099/mic.0.038257-0 